\title{
Submolecular Resolution Imaging of Molecules by Atomic Force Microscopy: The Influence of the Electrostatic Force
}

\author{
Joost van der Lit, ${ }^{1}$ Francesca Di Cicco, ${ }^{1}$ Prokop Hapala, ${ }^{2}$ Pavel Jelinek, ${ }^{2}$ and Ingmar Swart ${ }^{1, *}$ \\ ${ }^{1}$ Debye Institute for Nanomaterials Science, Utrecht University, P.O. Box 80.000, 3508 TA Utrecht, The Netherlands \\ ${ }^{2}$ Institute of Physics, Academy of Sciences of the Czech Republic Cukrovarnicka 10, 186253 Prague, Czech Republic
} (Received 10 December 2015; revised manuscript received 19 January 2016; published 3 March 2016)

\begin{abstract}
The forces governing the contrast in submolecular resolution imaging of molecules with atomic force microscopy (AFM) have recently become a topic of intense debate. Here, we show that the electrostatic force is essential to understand the contrast in atomically resolved AFM images of polar molecules. Specifically, we image strongly polarized molecules with negatively and positively charged tips. A contrast inversion is observed above the polar groups. By taking into account the electrostatic forces between tip and molecule, the observed contrast differences can be reproduced using a molecular mechanics model. In addition, we analyze the height dependence of the various force components contributing to the highresolution AFM contrast.
\end{abstract}

DOI: 10.1103/PhysRevLett.116.096102

The use of frequency modulated atomic force microscopy (FM AFM) as a tool to study molecules has grown tremendously over the past few years. The atomic structure of a single (unknown) molecule can now routinely be identified [1-3]. Even in the case of a complex mixture, the atomic structure of different molecules can be determined [4]. There is increasing interest in extracting quantitative information, such as bond orders, from AFM experiments [5-7].

Several papers have addressed the forces responsible for the submolecular contrast observed in AFM images of molecules [8-13]. It is now well established that the Pauli repulsion, i.e., the increase in the kinetic energy of electrons due to the overlap of electron clouds of the tip and molecule, is one of the most important force components [11]. In addition, van der Waals (vdW) interactions need to be taken into account. Finally, for molecule terminated tips, it is essential to account for the flexibility of the tip $[5,8,14]$. This flexibility is responsible for image distortions [15] and can enhance the contrast between nonbonded atoms to such an extent that it mimics an apparent bond $[8,9,16]$.

Thus far, mostly pure hydrocarbons were investigated, although recently also some images of molecules containing heteroatoms were published $[1,17-20]$. In the latter, the charge distribution can be highly nonhomogeneous. For molecules weakly bound to the substrate, the use of chemically passivated tips is essential to avoid accidental pickup of the molecule of interest $[2,14]$. When molecules are more strongly bound, also more reactive tips can be

Published by the American Physical Society under the terms of the Creative Commons Attribution 3.0 License. Further distribution of this work must maintain attribution to the author(s) and the published article's title, journal citation, and DOI. used [21,22]. Typically, chemical passivation is achieved by adsorbing a single $\mathrm{CO}$ molecule on the tip apex. It is known that a $\mathrm{CO}$ molecule adsorbed on a metal has a dipole moment [23,24], the size and direction of which are very sensitive to the adsorption geometry. Hence, when such a charged tip is used to image a polar molecule, it will experience an electrostatic force. Electrostatic forces are known to be important in the imaging of polar substrates $[23,25-28]$. This raises the question about what the influence of electrostatic forces is in AFM imaging of polar molecules with chemically passivated tips. Here, we investigate how the contrast in AFM images of a molecule with strongly polarized groups (carboxylic acid, triple bond) depends on the charge of the tip. The structure of the molecule bis-(para-benzoicacid) acetylene (BPBA) is shown in Fig. 1(a). The BPBA was deposited on a $\mathrm{Au}(111)$ surface, where it forms a self-assembled layer as shown in Fig. 1(b). We acquired high-resolution AFM images with $\mathrm{CO}$ and Xe terminated tips, which provide substantially different submolecular contrast. By comparing the experimental data to molecular mechanics simulations, this variation of the submolecular contrast is attributed to a negative (CO) and positive (Xe) charge on the tip $[23,29,30]$. In particular, we will show that the experimentally observed contrast can only be reproduced in simulations when electrostatic forces are taken into account [29]. Finally, we assess the height dependence of the different force contributions to the contrast.

BPBA molecules (synthesized according to Ref. [31]) were evaporated from a stainless-steel crucible using an ebeam evaporator (Focus $\mathrm{GmbH}$ ) operated at $0.5 \mathrm{~W}$ power onto a clean $\mathrm{Au}(111)$ crystal held at $5 \mathrm{~K}$ located in a lowtemperature STM/AFM (Omicron $\mathrm{GmbH}$ ). All images were recorded with a qPlus sensor $\left(f_{0}=21922 \mathrm{~Hz}\right.$, $\left.A_{p-p}=2 \AA, Q=30000\right)$. After verification that the 

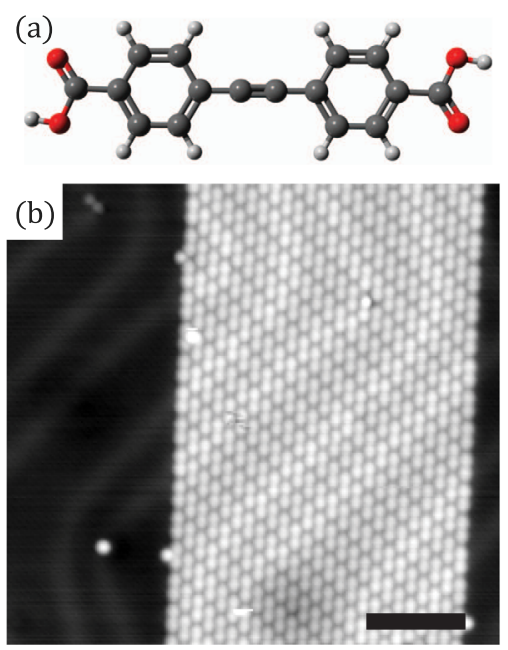
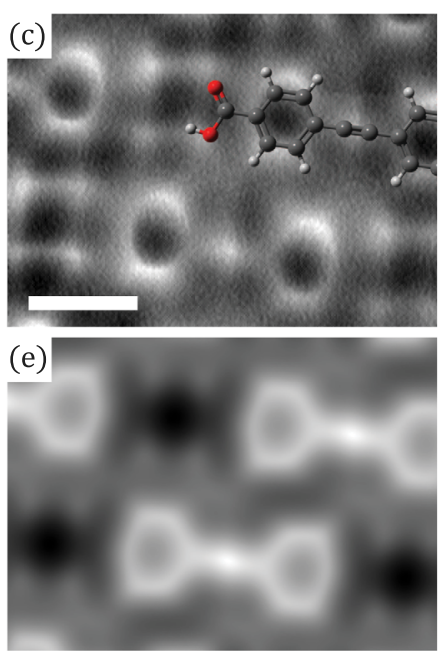
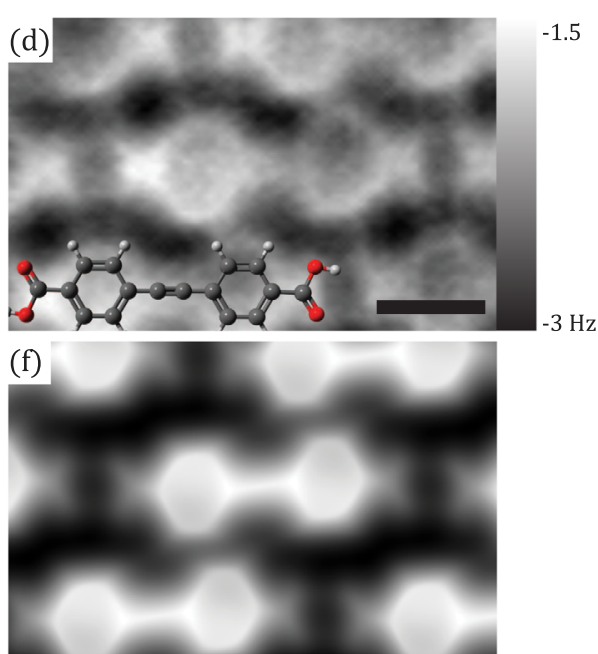

FIG. 1. BPBA molecules on Au(111) imaged with a $\mathrm{CO}$ and Xe tip. (a) Model of BPBA. (b) STM image of the self-assembled structure of BPBA on $\mathrm{Au}(111), 0.1 \mathrm{~V}, 10 \mathrm{pA}$, scale bar: $5 \mathrm{~nm}$. (c) Constant height $\Delta f$ image of BPBA recorded with a CO tip. Image recorded at $-1 \AA$ STM set point $(0.1 \mathrm{~V}, 10 \mathrm{pA})$, scale bar: $5 \AA$. (d) Constant height $\Delta f$ image of BPBA recorded with a Xe tip. Image recorded at $-2.55 \AA$ STM set point $(0.1 \mathrm{~V}, 10 \mathrm{pA})$, scale bar: $5 \AA$. (e) Simulated $\Delta f$ image for a CO terminated tip with a $Q_{\text {tip }}=-0.05 e^{-}$and $k_{x, y}=0.25 \mathrm{~N} / \mathrm{m}$. (f) Simulated $\Delta f$ image for a Xe tip with a $Q_{\text {tip }}=+0.3 e^{-}$and $k_{x, y}=0.25 \mathrm{~N} / \mathrm{m}$.

surface coverage was less than a monolayer, the crystal was allowed to heat to $300 \mathrm{~K}$ outside the microscope to allow the BPBA to self-assemble into a close-packed structure. The sample was placed back in the microscope after which $\mathrm{Xe}$ or $\mathrm{CO}$ was dosed onto the cold sample. $\mathrm{CO}$ and $\mathrm{Xe}$ terminated tips were prepared using standard procedures [32-36]. To minimize the influence of the vdW background, we studied self-assembled structures of BPBA. All AFM images were acquired at $0 \mathrm{~V}$ in constant-height mode. The height at which an image was recorded was set with respect to a STM set point (indicated in the text and figure captions). A negative height corresponds to a decrease in the tip-sample distance.

Figure 1(b) shows a STM overview of the self-assembled structure on $\mathrm{Au}(111)$. The well-known herringbone reconstruction of the $\mathrm{Au}(111)$ surface is clearly visible underneath the patches of molecules. Hence, the BPBA molecules interact weakly with the surface, in agreement with observations made for other molecules on $\mathrm{Au}(111)$ [37,38]. Constant-height AFM images acquired with CO and Xe terminated tips are shown in Figs. 1(c) and 1(d). We first discuss the contrast in the images acquired with a $\mathrm{CO}$ tip. Both oxygen atoms of the carboxylic acid group are imaged as bright dots. The carboxyl carbon atom (with partial positive charge) is not observed at this tip-sample distance with a CO tip, meaning it is still in the attractive regime. The electron-rich triple bond in the center of the molecule also appears as a bright protrusion when imaged with a CO tip, similar to what has been observed before [3]. Now we discuss how the contrast changes when a Xe terminated tip is used. The carbonyl carbon is imaged as more repulsive than the neighboring oxygen atoms (i.e., less negative frequency shift). In addition, the signature of the triple bond is no longer clearly resolved. Furthermore, the sizes of various features differ with different tips. Specifically, for these images the apparent distance between the oxygen atoms belonging to the same molecule is smaller with a Xe tip than with the CO tip $(1.6 \pm 0.2 \AA$ vs $2.8 \pm 0.2 \AA$ ); i.e., with a Xe tip the carboxylic acid group is imaged as a narrower forklike feature. Finally, the benzene rings appear larger when imaged with a Xe terminated tip (CO, $3.4 \pm 0.1 \AA$ vs Xe, $4.4 \pm 0.2 \AA$ ).

To shed light on the relative importance of the various force components, we performed extensive simulations using a periodic molecular mechanics model (AFMulator) [8-10,39], including the electrostatic force [29]. Briefly, the model calculates the tip-sample force $\left(F_{\mathrm{TS}}\right)$ via pairwise Lennard-Jones (LJ) potentials. Note that these potentials contain terms representing the vdW attraction and Pauli repulsion. In addition, electrostatic forces acting between an effective charge on the tip ( $\left.Q_{\text {tip }}\right)$ and electrostatic Hartree potential of the fully relaxed sample [29], as calculated using density-functional theory, are taken into account [40]. The main parameters in the simulations are the lateral force constant and the effective charge of the final atom of the tip denoted as $k_{x, y}$ and $Q_{\text {tip }}$, respectively. The result of the simulations for the Xe and CO tip with $k_{x, y}=0.25 \mathrm{~N} / \mathrm{m}$ and $Q_{\text {tip }}=-0.05 e^{-} /+0.3 e^{-}$for the $\mathrm{CO}$ and Xe terminated tips are shown in Figs. 1(e) and 1(f), respectively. These values for $k_{x, y}$ and $Q_{\text {tip }}$ are in agreement with previously reported values $[15,29,44]$. Our results are robust with respect to small changes in $Q_{\text {tip }}$ and $k_{x, y}$ (see the Supplemental Material [40]). All the features observed experimentally (contrast on and size difference in the carboxylic acid group, triple bond, size of benzene rings, discussed above) are reproduced well in the simulations. 


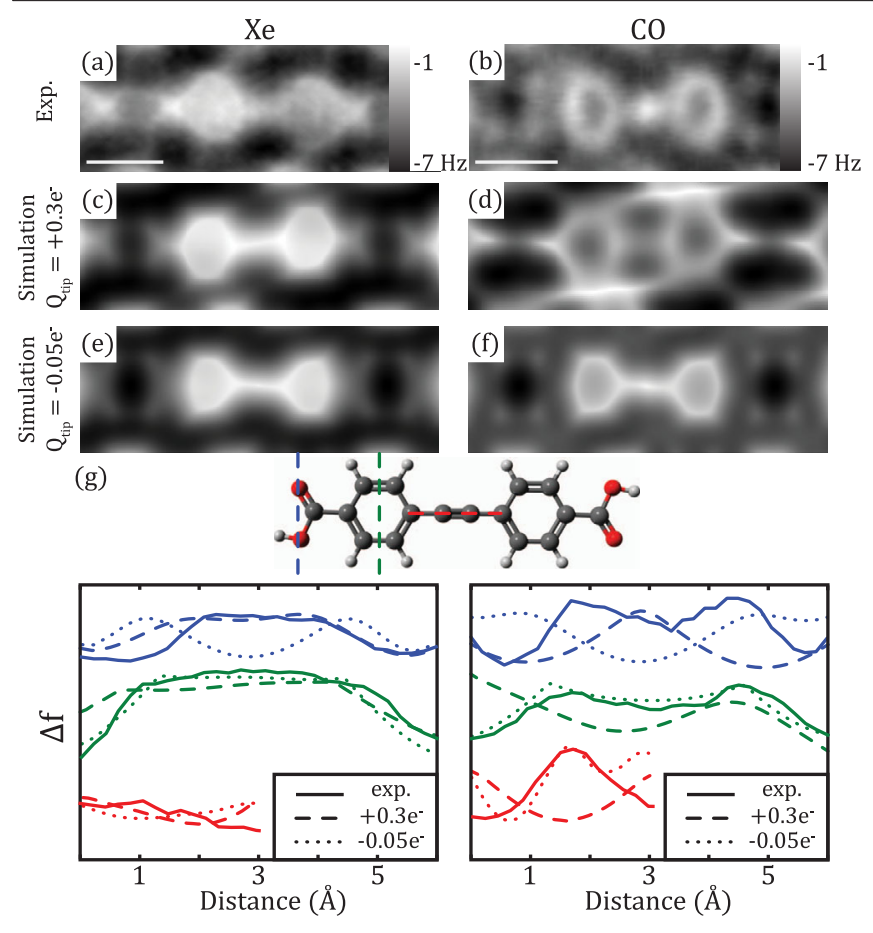

FIG. 2. Importance of tip charge in simulated $\Delta f$ images of BPBA in self-assembled structures. (a),(b) Constant-height $\Delta f$ images recorded with $\mathrm{Xe}$ and $\mathrm{CO}$ tips on a self-assembled layer of BPBA molecules $(-3 \AA$ and $-2.4 \AA$ STM set point $0.1 \mathrm{~V} / 10 \mathrm{pA})$. (c),(d) Simulations with tip charge: $Q_{\text {tip }}=$ $+0.3 e^{-}$for the $\mathrm{Xe}$ and $\mathrm{CO}$ terminated tips. (e),(f) Simulations with tip charge $Q_{\text {tip }}=-0.05 e^{-}$for the Xe and CO terminated tips. $k_{x, y}=0.25 \mathrm{~N} / \mathrm{m}$ in all calculations. (g) Line profiles over characteristic parts of the molecule (indicated in the ball-andstick model) from experimental data and simulations. Left panel: Xe. Right panel: CO. Scale bars: $5 \AA$.

To demonstrate the influence of the electrostatic force, we first calculated two positively charged tips with different LJ potentials: one corresponding to Xe [Fig. 2(c)] and the other one corresponding with $\mathrm{CO}$ [Fig. 2(d)] and compared them to experimental images [Figs. 2(a) and 2(b)]. The line profiles from characteristic parts of the molecule are shown in Fig. 2(g). Importantly, the effective positive charge on the $\mathrm{CO}$ tip gives a contrast that is comparable to that of the positively charged Xe tip. Namely, we observe a repulsive contrast on the carbonyl carbon and attractive contrast above the oxygen atoms and no clear signature of the triple bond. The additional image features in Fig. 2(d) between the molecules are due to a relatively large partial charge on the tip combined with the small atomic radius of the oxygen atom (1.66 $\AA$ vs $2.18 \AA$ for $\mathrm{O}$ and $\mathrm{Xe}$ ). The simulation with a negatively charged Xe tip shows similar contrast as the simulation with a negatively charged CO tip, with the exception of the triple bond. These findings conclusively demonstrate that the electrostatic force contribution is essential to understand the contrast in AFM images of polar molecules.

The submolecular contrast is, thus, driven by a combination of the attractive van der Waals forces, the repulsive
Pauli interaction, and the electrostatic interaction that can be both attractive and repulsive. At close distances, the shape of the features, e.g., the apparent position of atoms, strongly depends on the lateral relaxations of the flexible molecule or atom $(\mathrm{CO}, \mathrm{Xe})$ attached to the metallic tip apex. This implies that the presence of the electrostatic interaction not only changes the relative intensity of the observed AFM contrast over atoms or bonds, but it also affects the position of sharp contours due to an extra lateral relaxation of the flexible probe. Consequently, both the intensity and the shape of the features in submolecular AFM images acquired with functionalized tips with opposite effective charge (positive $\mathrm{Xe}$ and negative $\mathrm{CO}$ terminated tips) vary significantly, as shown in Fig. 2. The possibility to use distortions in AFM contrast to extract details about the tip charge and the spatial extent of the electrostatic force will be published elsewhere.

Since the electrostatic force, Pauli repulsion, and vdW attraction have a different distance dependence, it is important to establish the importance of each force component at different heights. To investigate the height dependence of these contributions, we acquired 150 constant-height $\Delta f$ images with a Xe terminated tip and with a spacing of $5 \mathrm{pm}$ starting from $-3.3 \AA$ with respect to a STM set point of $0.1 \mathrm{~V}$ and $10 \mathrm{pA}$. In addition, we simulated AFM images over the corresponding height range with and without the electrostatic force. Theory and experiment are brought in register by visual identification of the height at which contrast inversion occurs. At close tip-sample distances $(-3 \AA)$, the contrast is determined by the Pauli repulsion [Figs. 3(a)-3(c)]. The bright benzene rings are reproduced in calculations with and without the electrostatic force [Figs. 3(b) and 3(c)]. However, the slight asymmetric position of the benzene rings along the long-molecular axis, as well as the more narrow appearance of the carboxylic acid group is only reproduced in the calculation including the electrostatic force [cf. Figs. 3(a)-3(c)]. At intermediate heights $(-2.5 \AA)$, just after the onset of submolecular resolution, Pauli repulsion over the atoms results in submolecular contrast [Figs. 3(d)-3(f)]. From a comparison between Figs. 3(e) and 3(f), it is clear that the electrostatic force is essential to reproduce the experimentally observed repulsive contrast above the carbonyl carbon. We find that the benzene rings appear larger in simulations with a positively charged tip. Note that in addition to the effective charge of the tip, also the radius of the probe atom and the lateral stiffness of the tip affect the absolute size of the benzene rings. At a tip-sample distance just before the submolecular resolution $(-2.1 \AA)$, the contrast is very dependent on the electrostatic forces [Figs. 3(g)-3(i)]. A calculation with only $\mathrm{vdW}$ forces cannot reproduce the experimentally observed contrast above the triple bond (green arrows) and in the area between the molecules (red arrows). At even greater tip-sample separation $[-1.3 \AA$, Figs. $3(j)-3(1)]$, the 


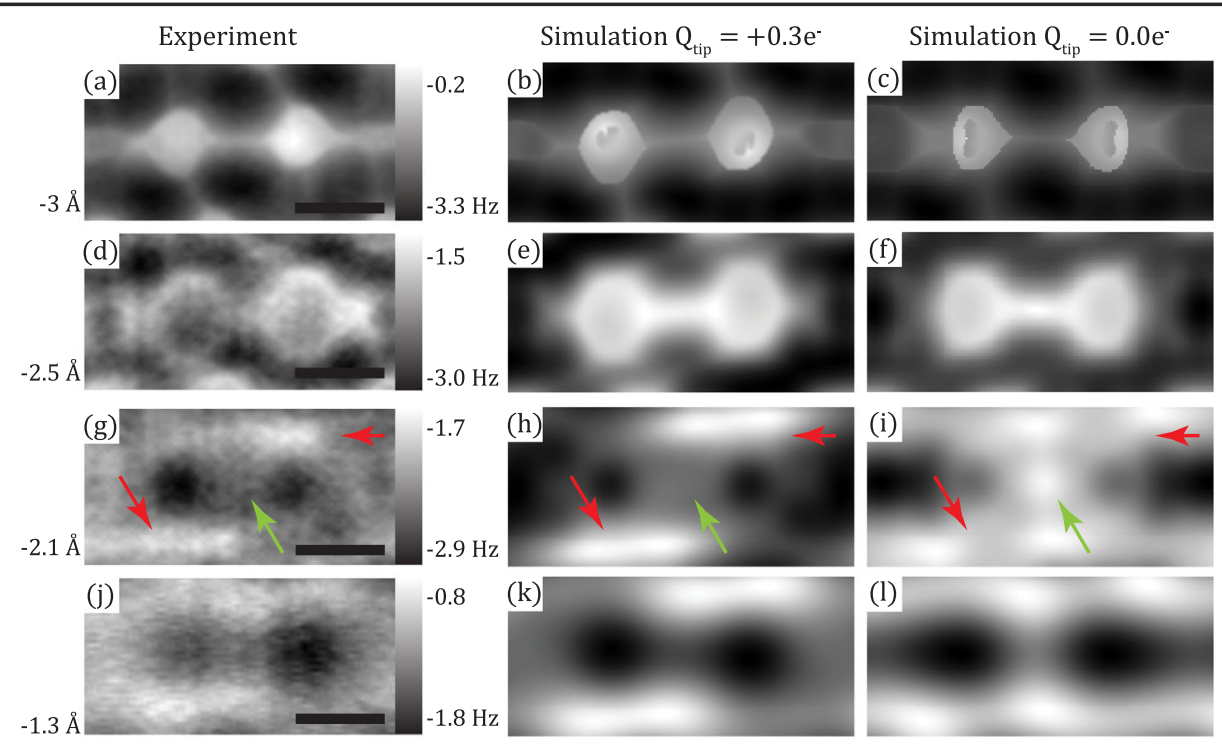

FIG. 3. Height dependence of $\Delta f$ images with a Xe tip compared to simulations with or without electrostatic force. (a)-(c) Experimental (a) and simulated AFM images with (b) and without (c) electrostatic force $\left(Q_{\text {tip }}=+0.3 e^{-}\right.$and $\left.0.0 e^{-}\right)$at $z=-3 \AA$. The tip height was set with respect to a STM set point of $0.1 \mathrm{~V}$ and 10 pA. (d)-(f) Same as (a)-(c) but at $z=-2.5 \AA$. (g)-(i) same as (a)-(c) but at $z=-2.1 \AA$. (j)-(1) Same as (a)-(c) but at $z=-1.3 \AA$. Simulations have the same $\Delta z$ between images as the experiment. All scale bars: $2.5 \AA$.

vdW attraction above the benzene rings dominates, and the molecule has a dumbbell shape in simulations with and without electrostatic interactions [Figs. 3(k) and 3(1)]. However, by including electrostatic forces, the agreement with experiment above and immediately next to the triple bond (additional attractive interaction) is improved. We conclude that taking into account electrostatic forces is essential to understand the observed contrast over a wide range of tip-sample distances, even at small distances where the Pauli repulsion dominates. We note here that the model currently does not incorporate the vdW attraction between the molecule and the bulk tip. This additional attractive contribution strongly depends on the macroscopic tip shape, something that is very difficult to control experimentally. However, this long-range vdW component does not generate the submolecular AFM contrast. Because of the relatively flat background in the close-packed selfassembled layer, the omission of the bulk tip will in the present case only result in a rigid offset of the calculated frequency shift values.

In the following, we assess the magnitude of each force contribution as calculated in the molecular mechanics simulations. Figure S2 [40] plots the electrostatic and $\mathrm{vdW}$ force-distance components calculated above characteristic molecular sites for both $\mathrm{CO}$ and $\mathrm{Xe}$ tips. The analysis reveals that the magnitude of the vdW component $(\approx 100 \mathrm{pN})$ is much larger than the electrostatic counterpart (only a few $\mathrm{pN}$ depending on the effective charge on the tip). The magnitude matches previously measured total tipsample forces on the order of $10-100 \mathrm{pN}$ for CO functionalized tips $[2,45]$. The sign of the electrostatic component (attractive or repulsive) varies depending on the charge distribution already at large distances, where the vdW component is mostly monotonically attractive, and the Pauli repulsion is negligible. This further supports the notion that the electrostatic force is essential to understand the contrast in AFM images of molecules at distances where the Pauli repulsion is still negligible.

In conclusion, we imaged strongly polarized BPBA molecules with negatively charged $\mathrm{CO}$ and positively charged Xe passivated tips. The combination of experimental high-resolution AFM imaging supported with theoretical modeling unveiled the impact of the electrostatic force acting between the surface and functionalized tip on the resulting submolecular contrast. We observed large differences in the $\Delta f$ contrast, which can be understood by taking into account the electrostatic contribution to the total force. These differences were observed at all tip-sample distances and contributed significantly to the submolecular AFM contrast. Thus, the electrostatic force plays a very important role in understanding submolecular AFM contrast on strongly polarized molecules.

We thank Peter Spijker and Martin Švec for discussions. This research was supported by the Netherlands Organization for Scientific Research (Chemical Sciences, Veni Grant No. 722.011.007) and the GAČR (Project No. 14-16963J).

*I.Swart@uu.nl

[1] L. Gross, F. Mohn, N. Moll, G. Meyer, R. Ebel, W. M. Abdel-Mageed, and M. Jaspars, Nat. Chem. 2, 821 (2010). 
[2] L. Gross, F. Mohn, N. Moll, P. Liljeroth, and G. Meyer, Science 325, 1110 (2009).

[3] D. G. de Oteyza, P. Gorman, Y.-C. Chen, S. Wickenburg, A. Riss, D. J. Mowbray, G. Etkin, Z. Pedramrazi, H.-Z. Tsai, A. Rubio, M. F. Crommie, and F. R. Fischer, Science 340, 1434 (2013).

[4] B. Schuler, G. Meyer, D. Peña, O. C. Mullins, and L. Gross, J. Am. Chem. Soc. 137, 9870 (2015).

[5] L. Gross, F. Mohn, N. Moll, B. Schuler, A. Criado, E. Guitian, D. Pena, A. Gourdon, and G. Meyer, Science 337, 1326 (2012).

[6] Y. Sugimoto, P. Pou, M. Abe, P. Jelinek, R. Pérez, S. Morita, and O. Custance, Nature (London) 446, 64 (2007).

[7] M. Ternes, C. P. Lutz, C. F. Hirjibehedin, F. J. Giessibl, and A. J. Heinrich, Science 319, 1066 (2008).

[8] P. Hapala, G. Kichin, C. Wagner, F. S. Tautz, R. Temirov, and P. Jelínek, Phys. Rev. B 90, 085421 (2014).

[9] S. K. Hämäläinen, N. van der Heijden, J. van der Lit, S. den Hartog, P. Liljeroth, and I. Swart, Phys. Rev. Lett. 113, 186102 (2014).

[10] M. P. Boneschanscher, S. K. Hämäläinen, P. Liljeroth, and I. Swart, ACS Nano 8, 3006 (2014).

[11] N. Moll, L. Gross, F. Mohn, A. Curioni, and G. Meyer, New J. Phys. 12, 125020 (2010).

[12] J. Zhang, P. Chen, B. Yuan, W. Ji, Z. Cheng, and X. Qiu, Science 342, 611 (2013).

[13] C.-S. Guo, M. A. Van Hove, X. Ren, and Y. Zhao, J. Phys. Chem. C 119, 1483 (2015).

[14] H. Mönig, D. R. Hermoso, O. Díaz Arado, M. Todorović, A. Timmer, S. Schüer, G. Langewisch, R. Pérez, and H. Fuchs, ACS Nano 10, 1201 (2016).

[15] M. Neu, N. Moll, L. Gross, G. Meyer, F. J. Giessibl, and J. Repp, Phys. Rev. B 89, 205407 (2014).

[16] S. P. Jarvis, M. A. Rashid, A. Sweetman, J. Leaf, S. Taylor, P. Moriarty, and J. Dunn, Phys. Rev. B 92, 241405 (2015).

[17] S. Kawai, S. Saito, S. Osumi, S. Yamaguchi, A. S. Foster, P. Spijker, and E. Meyer, Nat. Commun. 6, 8098 (2015).

[18] N. Pavliček, B. Fleury, M. Neu, J. Niedenführ, C. HerranzLancho, M. Ruben, and J. Repp, Phys. Rev. Lett. 108, 086101 (2012).

[19] F. Albrecht, J. Repp, M. Fleischmann, M. Scheer, M. Ondráček, and P. Jelínek, Phys. Rev. Lett. 115, 076101 (2015).

[20] B. Schuler, S.-X. Liu, Y. Geng, S. Decurtins, G. Meyer, and L. Gross, Nano Lett. 14, 3342 (2014).

[21] K. Iwata, S. Yamazaki, P. Mutombo, P. Hapala, M. Ondráček, P. Jelínek, and Y. Sugimoto, Nat. Commun. 6, 7766 (2015).

[22] A. Sweetman, S. P. Jarvis, P. Rahe, N. R. Champness, L. Kantorovich, and P. Moriarty, Phys. Rev. B 90, 165425 (2014).
[23] M. Schneiderbauer, M. Emmrich, A. J. Weymouth, and F. J. Giessibl, Phys. Rev. Lett. 112, 166102 (2014).

[24] A. Schwarz, A. Köhler, J. Grenz, and R. Wiesendanger, Appl. Phys. Lett. 105, 011606 (2014).

[25] F. J. Giessibl, Phys. Rev. B 45, 13815 (1992).

[26] G. Teobaldi, K. Lämmle, T. Trevethan, M. Watkins, A. Schwarz, R. Wiesendanger, and A. L. Shluger, Phys. Rev. Lett. 106, 216102 (2011).

[27] F. Bocquet, L. Nony, and C. Loppacher, Phys. Rev. B 83, 035411 (2011).

[28] R. Hoffmann, D. Weiner, A. Schirmeisen, and A. S. Foster, Phys. Rev. B 80, 115426 (2009).

[29] P. Hapala, R. Temirov, F. S. Tautz, and P. Jelinek, Phys. Rev. Lett. 113, 226101 (2014).

[30] L. Gross, B. Schuler, F. Mohn, N. Moll, N. Pavliček, W. Steurer, I. Scivetti, K. Kotsis, M. Persson, and G. Meyer, Phys. Rev. B 90, 155455 (2014).

[31] H. Dibowski and F. P. Schmidtchen, Tetrahedron 51, 2325 (1995).

[32] D. M. Eigler, C. P. Lutz, and W. E. Rudge, Nature (London) 352, 600 (1991).

[33] B. Neu, G. Meyer, and K.-H. Rieder, Mod. Phys. Lett. B 09, 963 (1995).

[34] A. Yazdani, D. M. Eigler, and N. D. Lang, Science 272, 1921 (1996).

[35] G. Kichin, C. Weiss, C. Wagner, F. S. Tautz, and R. Temirov, J. Am. Chem. Soc. 133, 16847 (2011).

[36] L. Bartels, G. Meyer, and K. Rieder, Appl. Phys. Lett. 71, 213 (1997).

[37] J. van der Lit, M. P. Boneschanscher, D. Vanmaekelbergh, M. Ijäs, A. Uppstu, M. Ervasti, A. Harju, P. Liljeroth, and I. Swart, Nat. Commun. 4, 2023 (2013).

[38] W.-H. Soe, C. Manzano, A. De Sarkar, N. Chandrasekhar, and C. Joachim, Phys. Rev. Lett. 102, 176102 (2009).

[39] https://github.com/ProkopHapala/ProbeParticleModel.

[40] See the Supplemental Material at http://link.aps.org/ supplemental/10.1103/PhysRevLett.116.096102, which includes Refs. [41-43], for a more detailed description of the model, including the parameters used to produce the pair potentials.

[41] G. Kresse and J. Furthmüller, Phys. Rev. B 54, 11169 (1996).

[42] J. P. Perdew, J. A. Chevary, S. H. Vosko, K. A. Jackson, M. R. Pederson, D. J. Singh, and C. Fiolhais, Phys. Rev. B 46, 6671 (1992).

[43] P. E. Blöchl, Phys. Rev. B 50, 17953 (1994).

[44] A. J. Weymouth, T. Hofmann, and F. J. Giessibl, Science 343, 1120 (2014).

[45] M. P. Boneschanscher, J. van der Lit, Z. Sun, I. Swart, P. Liljeroth, and D. Vanmaekelbergh, ACS Nano 6, 10216 (2012). 